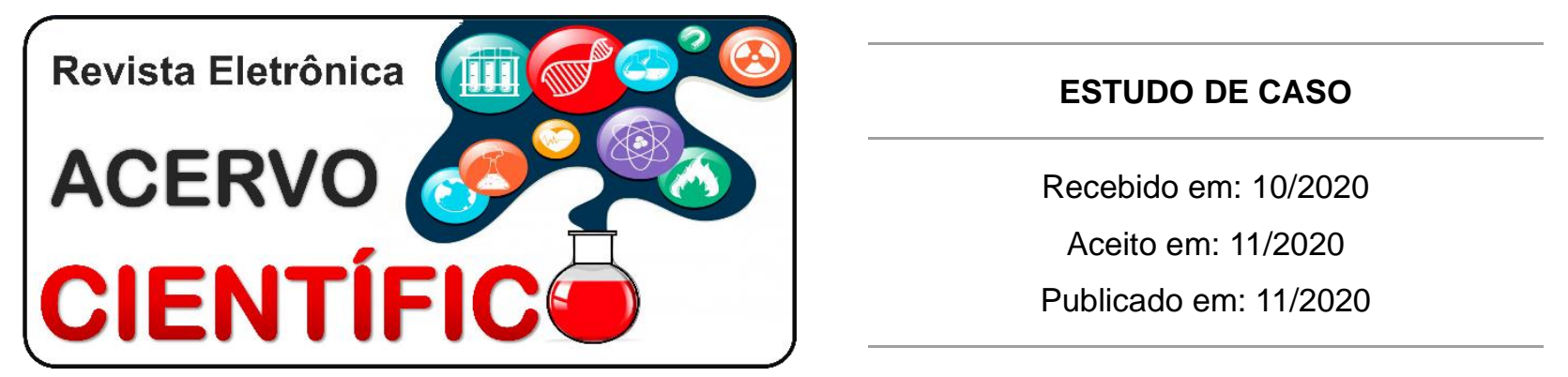

\title{
Laceração hepática grau II: intervenção cirúrgica após instabilidade hemodinâmica - relato de caso
}

\author{
Grade II hepatic laceration: surgical intervention after hemodynamic instability - case \\ report
}

Laceración hepática de grado II: intervención quirúrgica tras inestabilidad hemodinâmica reporte de caso

Henrique Amorim Santos ${ }^{1}$, Isabella Amorim Santos², Amanda Karolyne Batista Ferreira ${ }^{1}$, Marcella Gonçalves de Laia1', Júlia Martins Roriz¹, Ana Carolina Cunha Leal', Guilherme Rodrigues Oliveira ${ }^{1}$, Júlia Carmo Vilela¹.

\begin{abstract}
Resumo: A lesão hepática traumática é prevalente e possui altas taxas de morbimortalidade. A conduta terapêutica varia de acordo com as características da lesão e com os parâmetros clínicos do paciente. As lesões hepáticas são classificadas em seis graus de gravidade, que auxiliam na decisão terapêutica. Lesões hepáticas de baixo grau (graus I, II e III) são frequentemente tratadas com sucesso por manejo não cirúrgico. Esse artigo buscou relatar o caso de um paciente do sexo masculino, 52 anos, com histórico de trauma abdominal contuso após acidente automobilístico, admitido no pronto-socorro inicialmente estável. Evoluiu após algumas horas, entretanto, com instabilidade hemodinâmica, sendo indicada laparotomia de emergência. Paciente apresentava lesão hepática grau II, que, contudo, é manejada normalmente com tratamento não operatório. O tratamento cirúrgico evoluiu com boa recuperação do paciente, que teve alta hospitalar após doze dias. É essencial, portanto, o acompanhamento rigoroso da evolução do paciente com trauma hepático a fim de optar pela melhor conduta terapêutica.
\end{abstract}

Palavras-chave: Fígado, Ferimentos e lesões, Laparotomia.

\begin{abstract}
Traumatic liver injury is prevalent and has high rates of morbidity and mortality. The therapeutic approach varies according to the characteristics of the lesion and the patient's clinical parameters. Liver injuries are classified into six degrees of severity, which help in the therapeutic decision. Low-grade liver injuries (grades I, II and III) are often successfully treated by non-surgical management. This article sought to report the case of a 52-year-old male patient with a history of blunt abdominal trauma after a car accident, admitted initially stable to the emergency room. It evolved after a few hours, however, with hemodynamic instability, and emergency laparotomy was indicated. The patient had grade II liver damage, which, however, is usually managed with non-operative treatment. Surgical treatment evolved with good recovery of the patient, who was discharged after twelve days. Therefore, it is essential to strictly monitor the evolution of the patient with liver trauma in order to choose the best therapeutic approach.
\end{abstract}

Keywords: Liver, Wounds and injuries, Laparotomy.

1 Universidade Federal do Triângulo Mineiro (UFTM), Uberaba - MG. E-mail: henriqueamorims@gmail.com

2 Universidade de Franca (UNIFRAN), Franca - SP. 
Resumen: La lesión hepática traumática es frecuente y tiene altas tasas de morbilidad y mortalidad. El abordaje terapéutico varía según las características de la lesión y los parámetros clínicos del paciente. Las lesiones hepáticas se clasifican en seis grados de gravedad, que ayudan en la decisión terapéutica. Las lesiones hepáticas de bajo grado (grados I, II y III) a menudo se tratan con éxito mediante un tratamiento no quirúrgico. Este artículo buscó reportar el caso de un paciente masculino de 52 años con antecedente de traumatismo abdominal cerrado tras accidente automovilístico, ingresado en urgencias inicialmente estable. Sin embargo, evolucionó a las pocas horas con inestabilidad hemodinámica, por lo que se indicó laparotomía urgente. El paciente tenía daño hepático de grado II, que, sin embargo, generalmente se trata con tratamiento no quirúrgico. El tratamiento quirúrgico evolucionó con buena recuperación del paciente, que fue dado de alta a los doce días. Por tanto, es fundamental un seguimiento estricto de la evolución del paciente con traumatismo hepático para elegir el mejor abordaje terapéutico.

Palabras clave: Hígado, Heridas y traumatismos, Laparotomía.

\section{INTRODUÇÃO}

O trauma abdominal pode ser classificado em dois tipos diferentes: penetrante ou contuso. A literatura aponta que, aproximadamente, $15 \%$ a $20 \%$ das lesões abdominais referem-se a trauma hepático, e que $80 \%$ a $90 \%$ desses traumas são lesões hepáticas contusas (YU WY, et al., 2016). É visto que $72 \%$ das lesões traumáticas de fígado ocorrem devido ao mecanismo de colisões de veículo (MALUTTA SS, et al., 2012). Um dos órgãos intra-abdominais mais acometidos em consequência do trauma é o fígado, uma vez que seu tamanho e sua localização predispõem para a ocorrência de lesões, sendo constatado na literatura que $20 \%$ das vítimas de trauma contuso sofrem lesão hepática (PETROWSKY H, et al., 2012). Nesses casos, as lesões podem ser decorrentes do impacto direto, da compressão entre o rebordo costal direito e a coluna vertebral e da força de desaceleração, sendo o lobo direito do fígado a região mais atingida durante a lesão abdominal, uma vez que essa é a porção do parênquima hepático mais volumosa (ROMANO L, et al., 2004).

A lesão isolada do fígado representa somente $10 \%$ dos casos de indivíduos que sofreram o trauma, ou seja, na grande maioria dos casos há lesões de outros órgãos e outras vísceras concomitantemente. (CHMATAL P, et al., 2008). Sabe-se que a taxa de mortalidade para pacientes com trauma hepático é maior naqueles que foram vítimas de trauma contuso $(40 \%)$ em relação àqueles que sofreram trauma penetrante $(12,5 \%)$, sendo que a explicação se dá pela maior taxa de lesões cranianas associadas ao trauma contuso, como consequência de trauma cranioencefálico grave (KALIL M, et al., 2016). Tal fato ressalta a gravidade desse tipo de trauma, que mesmo diante de avanços tecnológicos tanto dos diagnósticos, quanto dos tratamentos, além da sistematização do atendimento ao politraumatizado proposta pelo Advanced Trauma Life Support (ATLS), ainda possui taxas de morbimortalidade elevadas (KALIL M, et al., 2016). Esse cenário alarmante reflete em um grave problema de saúde pública, não só pelos problemas sociais, mas também pelos custos econômicos significativos, tendo em vista que a grande maioria das vítimas são indivíduos em idade produtiva (KALIL M, et al., 2016).

No que diz respeito à gravidade dos traumas hepáticos, é possível graduá-los de I a VI, de acordo com a Associação Americana de Cirurgia do Trauma (AACT). Essa classificação baseia-se no tipo e na descrição anatômica da lesão, em que aqueles que são classificados como graus I-III não necessitam de tratamento cirúrgico. Entretanto, em casos de instabilidade hemodinâmica, essa indicação de tratamento conversador poderá ser alterada. Portanto, para a adequada abordagem do paciente com trauma hepático é necessário não somente fazer a classificação anatômica da AACT, mas também a análise do perfil hemodinâmico e das demais lesões apresentadas pela vítima (COCCOLINI F, et al., 2016).

Para realizar o diagnóstico do trauma hepático a anamnese e o exame físico do paciente são de grande importância, em que, frequentemente, constata-se histórico de trauma contuso ou penetrante no abdome, além de manifestações clínicas típicas. Ademais, o exame de imagem é amplamente utilizado, sendo a tomografia computadorizada (TC), juntamente com o ultrassom, o método mais valioso para avaliar o 
trauma abdominal (YU WY, et al., 2016). A ultrassonografia FAST é um exame de suma importância na sala de emergência, visto que, além de ser realizada à beira do leito, possui alta sensibilidade tanto para o diagnóstico de hemoperitônio em pacientes hemodinamicamente instáveis, quanto para a identificação de lesões hepáticas, o que torna esse um exame ímpar no manejo do paciente vítima de trauma hepático. (RADWAN MM e ABU-ZIDAN FM, 2006).

De acordo com a literatura, os pacientes com trauma hepático que apresentarem instabilidade do estado hemodinâmico, evisceração, lesões internas associadas ou empalação deverão ser indicados à laparatomia de emergência, sendo constatado que o manejo cirúrgico é o tratamento de escolha em $20 \%$ dos casos. Em situações em que o paciente não pode ser estabilizado pela ressuscitação com fluidos ou sofre um choque grave, deve-se determinar prontamente a ordem de gravidade da doença hepática e prosseguir com laparotomia exploratória (LETOUBLON C, et al., 2016; YU WY, et al., 2016). Tendo em vista a redução da mortalidade e o tratamento adequado da laceração hepática, são fatores protetores o diagnóstico precoce, a avaliação precisa, a ativa resistência ao choque, o plano de tratamento ideal e a preservação da função do órgão (YU WY, et al., 2016).

Nessa perspectiva, o presente estudo tem como objetivo relatar o caso de um paciente vítima de um trauma abdominal contuso, com consequente lesão hepática grau II, e, posteriormente, discutir a indicação do manejo cirúrgico para esse paciente.

\section{DETALHAMENTO DO CASO}

Paciente masculino, 52 anos, admitido no pronto-socorro devido trauma abdominal contuso após acidente automobilístico. Apresentava-se estável hemodinamicamente, sem taquicardia. Em exame FAST (Avaliação Focalizada com Sonografia para Trauma), notou-se líquido no espaço de Morris e periesplênico em moderada intensidade. Após 7 horas da admissão, paciente evoluiu com palidez cutânea, sudorese pegajosa, náuseas, vômitos, dor abdominal importante com descompressão brusca dolorosa, pressão arterial de $83 \times 59 \mathrm{mmHg}$, além de queda de hemoglobina $(\mathrm{Hb}) 9,1$ para $8,5 \mathrm{~g} / \mathrm{dL}$.

Indicada laparotomia exploradora de urgência. No inventário da cavidade, encontrou-se moderada quantidade de sangue coletado em abdome superior, identificada lesão contusa em mesocólon transverso (com hematoma), em retroperitôneo e na segunda porção do duodeno. Verificou-se uma laceração hepática grau II de $6 \mathrm{~cm}$ de extensão com realização de hemostasia com o hemostático absorvível Gelfoam. Em pósoperatório paciente apresentou-se pálido e com anemia $(\mathrm{Hb}=7 \mathrm{~g} / \mathrm{dL})$, sendo transfundido dois concentrados de hemácias.

Na admissão o paciente também apresentou lesão de partes moles com exposição óssea em região anterior da tíbia proximal direita, em que foram identificados, pela tomografia computadorizada, fratura cominutiva metaepifisária proximal da tíbia com extensão intra-articular junto ao platô lateral e disjunção metadiafisária (Schatzker VI), além de fratura do colo da fíbula. Paciente foi encaminhado e seguiu em acompanhamento pela ortopedia. Também foi tratado hemotórax bilateral, sendo submetido à drenagem intercostal direita e tratamento conservador à esquerda. Paciente evoluiu sem mais intercorrências, com alta hospitalar no décimo segundo dia pós-laparotomia de emergência.

\section{DISCUSSÃO}

O trauma hepático tem uma maior prevalência em indivíduos do sexo masculino, coincidente com o caso em questão, devido principalmente a fatores comportamentais que aumentam o risco de acidentes nesse grupo populacional (KALIL M e AMARAL IMAI, 2016). Além disso, estudos demonstram que mais de $80 \%$ dos pacientes com trauma hepático possuem lesões intra e extra-abdominais associadas (KALIL M e AMARAL IMAI, 2016). No paciente em questão, verificou-se lesões intra-abdominais no cólon e duodeno superior e lesões extra-abdominais no tórax, com hemotórax bilateral, e fraturas ósseas, fatos que corroboraram para o maior agravamento do quadro.

O diagnóstico do trauma hepático em si é não é uma tarefa árdua. Assim como foi descrito no caso, os pacientes, em sua maioria, possuem história de trauma contuso ou penetrante, atrelada a manifestações 
clínicas características, como dor abdominal no quadrante superior direito (com possível irradiação para o ombro direito), náuseas, vômitos, sede, choque hipovolêmico e peritonite. Estes sinais e sintomas, atrelados ao correto uso de exames de imagem, garantem um melhor diagnóstico dos traumas hepáticos (YU WT, et al., 2016). Embora a tomografia computadorizada (TC) seja o método mais utilizado para o diagnóstico de lesões hepáticas, por sua capacidade de revelar pequenos danos hepáticos ocultos e avaliar seu sangramento (BADGER SA, et al., 2009; FU CJ, et al., 2015), no caso relatado, o exame de escolha foi a ultrassonografia abdominal do tipo FAST, a qual é adequada para pacientes hemodinamicamente instáveis, que não toleram a TC e que necessitam de um diagnóstico rápido acerca de uma possível hemorragia abdominal (YU WT, et al., 2016).

Desse modo, os aspectos mais relevantes e preliminares são o resgate e a avaliação precoces. Principalmente em pacientes hemodinamicamente instáveis, é de suma importância a determinação da gravidade da lesão hepática para a determinação da escolha terapêutica, não cirúrgica ou cirúrgica, e prosseguimento com a laparotomia exploratória oportuna e tratamento adequado (YU WT, et al., 2016).

A lesão hepática do relato apresentado, classificada como grau II, é caracterizada pela AACT por hematoma subcapsular não expansivo que acomete de 10 a $50 \%$ da superfície ou hematoma intraparenquimal com menos de $10 \mathrm{~cm}$ de extensão e laceração de $1 \mathrm{a} 3 \mathrm{~cm}$ de profundidade com menos de $10 \mathrm{~cm}$ de extensão (COCCOLINI F, et al., 2016). De acordo com essa classificação, esse tipo de lesão é classificada como lesão menor e junto ao grau I constituem uma importante prevalência nos traumas hepáticos, cerca de 80 a 90\% (YU WT, et al., 2016).

A maior parte dos pacientes admitidos na emergência com lesões hepáticas dos tipos grau I, II e III, aproximadamente $80 \%$ deles, são tratados com sucesso por manejo não cirúrgico. Porém, faz-se necessário uma investigação minuciosa não apenas da classificação da lesão hepática, mas também do status hemodinâmico, das condições clínicas e da presença de outros tipos de lesões nos pacientes vítimas de trauma hepático (COCCOLINI F, et al., 2016). De acordo com o ATLS, a definição de paciente instável é aquele com pressão arterial sistólica inferior a $90 \mathrm{mmHg}$; batimentos cardíacos superiores a $120 \mathrm{bpm}$; associada a evidências de vasoconstrição cutânea, como pele fria, pegajosa e com enchimento capilar diminuído; nível alterado de consciência e/ou dispneia. Este quadro clínico instável, presente no caso em questão, foi determinante para a escolha de tratamento cirúrgico em detrimento da classificação da AACT.

A modalidade de tratamento não cirúrgico tem sido cada vez mais utilizada, com $80 \%$ dos traumas de fígado conduzidos dessa maneira. Nos casos de grau I a III que são conduzidos de forma conservadora, obtêm-se bons resultados, com baixas taxas de complicações e mortalidade. A estabilidade hemodinâmica é a base da terapia não operatória, ao passo que este manejo é feito com base na premissa de que os pacientes não têm outros órgãos lesados, principalmente nenhum dano intestinal (YU WT, et al., 2016). Logo, a presença de instabilidade hemodinâmica e/ou peritonite faz o tratamento não operatório ser contraindicado independente da extensão da lesão hepática. Apesar dessa modalidade de tratamento ter grande sucesso, algumas complicações podem ocorrer, Coccolini F, et al. (2016), relatam as mais frequentes como hemorragias, síndrome de compartimento abdominal, infecções que podem evoluir para abscessos, complicações da vesícula biliar e necrose hepática, por isso se faz necessária a monitorização dos pacientes e acompanhamento laboratorial.

No entanto, no relato apresentado, optou-se pela intervenção cirúrgica devido à deterioração hemodinâmica, delimitada sobretudo pela hipotensão arterial e sinais de vasoconstrição (palidez cutânea e sudorese pegajosa) associados ao quadro clínico complexo de politrauma com lesões ortopédicas, danos intestinais no duodeno e no cólon além de hemotórax bilateral. Para os pacientes com instabilidade hemodinâmica, quanto mais rápida a conduta cirúrgica, maiores chances de se obter um bom resultado e evitar morbimortalidade (YU WT, et al., 2016). A laparotomia de emergência está cada vez mais rara, mas, quando realizada, seu objetivo principal é interromper o sangramento com a realização da hemostasia. Esta, pode ser realizada de forma efetiva de diversas maneiras como a compressão isolada do local, uso de eletrocautério, entre outras, sendo o uso de agentes hemostáticos como o Gelfoam optado no tratamento do paciente em questão (COCCOLINI F, et al., 2016). 
A laparotomia de emergência realizada no paciente deste caso, foi feita de forma eficaz e sem agravamentos no pós operatório. Apesar disso, vale ressaltar que essa modalidade de tratamento pode ocasionalmente ter complicações, assim como no tratamento não operatório. Normalmente, essas complicações estão relacionadas a gravidade e extensão da lesão hepática, associado ao perfil de instabilidade hemodinâmica do paciente, sendo as principais: sangramento tardio, fístula biliar e formação de abcessos abdominais, ou cistos e necrose do parênquima hepático (YU WT, et al., 2016). Quando há presença de alguma complicação, a drenagem pode ser realizada, ou em casos mais graves de sangramentos intensos e paciente instável, pode-se optar pela reoperação. Por conta disso, a observação no pós-operatório é importante para detectar essas complicações e por conseguinte realizar um tratamento eficaz (YU WT, et al., 2016). Neste caso, o paciente permaneceu no hospital por doze dias, tratou-se os demais traumas e lesões e com a melhora foi possível a alta.

A lesão hepática traumática é prevalente e possui altas taxas de morbimortalidade, representando um grave problema de saúde pública. Apesar de lesões de baixo grau (I-III) serem frequentemente manejadas de forma não operatória, a lesão de grau II apresentada teve indicação cirúrgica. O acompanhamento da evolução clínica e laboratorial de paciente com trauma hepático é essencial, visto que, a exemplo do caso relatado, em um primeiro momento o paciente estava estável, mas evoluiu com instabilidade hemodinâmica, necessitando de intervenção cirúrgica com laparotomia de emergência, indicada em um quinto dos casos de trauma hepático. Ademais, a avaliação multidisciplinar do paciente politraumatizado torna-se imprescindível para que a equipe decida a conduta mais eficiente a ser tomada.

\section{REFERÊNCIAS}

1. AMERICAN COLLEGE OF SURGEONS. Advanced trauma life support. 10th edition. Chicago: American College of Surgeons, Committee on Trauma, 2018; 474p.

2. BADGER SA, et al. Management of Liver Trauma. World Journal Of Surgery, 2009; 33(12): 2522-2537.

3. CHMATAL P, et al. Liver trauma usually means management of multiple injuries: analysis of 78 patients. Int Surg, 2008; 93(2):72-7.

4. COCCOLINI F, et al. WSES classification and guidelines for liver trauma. World J Emerg Surg, 2016;11(1):50.

5. FU CJ, et al. Computed tomography arterial portography for assessment of portal vein injury after blunt hepatic trauma. Diagnostic And Interventional Radiology, 2015; 21(5): 361-367

6. KALIL M, AMARAL IMAI. Epidemiological evaluation of hepatic trauma victims undergoing surgery. Rev Col Bras Cir, 2016;43(1):22-7.

7. LETOUBLON C, et al. Management of blunt hepatic trauma. Journal of Visceral Surgery, 2016;153(4):33-43.

8. MALUTTA SS, et al. Trauma hepático: principais características e importância do tratamento conservador. Panamerican Journal of Trauma, Critical Care \& Emergency Surgery, 2012;1(3):188-90.

9. PETROWSKY H, et al. A quarter century experience in liver trauma: a plea for early computed tomography and conservative management for all hemodynamically stable patients. World J Surg, 2012;36(2):247-54.

10. RADWAN MM, ABU-ZIDAN FM. Focussed Assessment Sonograph Trauma (Fast) and CT scan in blunt abdominal trauma: surgeon's perspective. Afr Health Sci, 2006;6(3):187-90.

11. ROMANO L, et al. Hepatic trauma: CT findings and considerations based on our experience in emergency diagnostic imaging. European Journal of Radiology, 2004; 50(1):59-66.

12. STALHSCHMIDT CMM, et al. Controle de danos no trauma abdominal e lesões associadas: experiência de cinco anos em um serviço de emergência. Rev Col Bras Cir, 2006;33(4):215-9.

13. YU WY, et al. Treatment strategy for hepatic trauma. Chinese Journal of Traumatology, 2016;19(3):168-71. 